East African Medical Journal Vol. 87 No. 1 January 2010

MODE OF DELIVERY DECISIONS AMONG HIV -INFECTED MOTHERS AT AN URBAN MATERNITY HOSPITAL IN KENYA

J. H. Beard, MD, Department of Obstetrics and Gynaecology, College of Health Sciences, University of Nairobi, P.O. Box 19676 - 00202, Nairobi, Kenya and Yale University School of Medicine, New Haven, CT, USA, S. W. Ndegwa, MBChB, Department of Obstetrics and Gynaecology, College of Health Sciences, University of Nairobi, P. O. Box 19676 - 00202, Nairobi, C. Farquhar, MD, MPH, Department of Medicine and Epidemiology, University of Washington, Seattle, USA, J. O. Ong'ech, MBChB, MMed, Department of Obstetrics and Gynaecology, F. Govedi, MBChB, MMed, Pumwani Maternity Hospital, Nairobi, Kenya and J. N. Kiarie, MBChB, MMed, MPH, Department of Obstetrics and Gynaecology, College of Health Sciences, University of Nairobi, P. O. Box 19676 - 00202, Nairobi, Kenya

Request for reprints to: Dr. J. H. Beard, 740 Parnassus Apt 14, San Francisco, CA 94122 USA

\title{
MODE OF DELIVERY DECISIONS AMONG HIV -INFECTED MOTHERS AT AN URBAN MATERNITY HOSPITAL IN KENYA
}

\author{
J. H. BEARD, S. W. NDEGWA, C. FARQUHAR, J. O. ONG'ECH, F. GOVEDI and J. N. KIARIE
}

\begin{abstract}
Objectives: To quantify the use of elective Caesarean section (ECS) for prevention of mother-to-child transmission of HIV (PMTCT) at an urban Kenyan maternity hospital, to describe mode of delivery decision making among HIV positive women, and to understand patient knowledge and attitudes regarding ECS for PMTCT.

Design: Descriptive cross-sectional study.

Setting: Pumwani Maternity Hospital, Nairobi, Kenya.

Subjects: Two hundred and fifty postpartum HIV-infected women.

Main outcome measures: ECS delivery rate, correlates of mode of delivery decisions and ECS for PMTCT knowledge and attitudes

Results: The rate of delivery by ECS for PMTCT was $4.0 \%$ (10/250), though $13.6 \%$ (34/250) planned this mode of delivery. Patient education regarding ECS for PMTCT was limited, and $64 \%(160 / 250)$ of participants had never heard of ECS. Planning ECS for PMTCT was positively correlated with attending clinic at PMH (OR=9.12, 95\% CI: 2.94-28.28, p<0.001), knowledge of ECS (OR=27.22, 95\% CI: 5.04-148.20, $\mathrm{p}<0.001)$ and having a history of abdominal surgery $(\mathrm{OR}=30.96,95 \% \mathrm{CI}: 6.32-205.02, \mathrm{p}<0.001)$. Delivering by ECS was associated with planning this mode of delivery $(\mathrm{OR}=19.52,95 \%$ CI: 3.69-103.23, p<0.001). Planning but not delivering by ECS was mostly due to labour before scheduled ECS $(55.6 \%, 15 / 27)$ or poor patient understanding of the intervention $(29.6 \%, 8 / 27)$. After education on ECS for PMTCT, $48.0 \%$ (120/250) of participants would consider elective Caesarean section if offered, though cost represented a significant barrier to acceptability.

Conclusions: Knowledge and utilisation of ECS for PMTCT are limited and varied in this patient population. ECS may be an acceptable mode of delivery for some Kenyan women, especially if the burden of cost is removed. A clear policy on ECS counselling and utilisation is urgently needed to ensure consistent and appropriate use of this PMTCT intervention in Kenya.
\end{abstract}

\section{INTRODUCTION}

Each day, 1800 children are newly infected with HIV worldwide, the vast majority of them in sub-Saharan Africa (1). Generally, infected children acquire HIV from their mothers during pregnancy, at time of delivery, or through breastfeeding. In Nairobi, Kenya, HIV prevalence among antenatal women is nearly $11 \%$, thus mother-to-child HIV transmission represents a substantial threat to paediatric health in the country (1).
Established in 2004, the prevention of motherto-child transmission of HIV (PMTCT) programme at Nairobi's Pumwani Maternity Hospital (PMH) currently targets vertical transmission of HIV by providing free services to infected women and their children, including antenatal HIV counselling and testing, antiretroviral (ARV) prophylaxis, safe obstetric practices during labour and delivery, education regarding safe infant feeding options, replacement feeding with infant formula at no cost, and HIV DNA PCR testing of children. Despite these interventions, 
rates of HIV transmission in Nairobi hospitals range from 6-16\% of infants born to infected women (compared to rates of less than $1 \%$ in developed countries) according to recent programme evaluation data (KiarieJ. N.etal.Effectiveness of a comprehensive PMTCT Programme. Poster Presentation for International AIDS Conference, 2006).

Elective Caesarean section (ECS), defined as Caesarean section before labour and rupture of membranes, is a PMTCT tool used widely in Western Europe and the United States $(2,3)$. The appropriateness of ECS for PMTCT in "resourcepoor" settings, however, is unclear in light of limited availability, cost and risk of post-partum complications (4). Although World Health Organisation (WHO) policy states that routine use of ECS for PMTCT in resource-poor settings is currently unrealistic, PMTCT guidelines in some sub-Saharan Africa countries suggest that ECS may be recommended if labour is expected to be prolonged, when complications may increase vertical transmission (e.g. placenta previa), in breech presentation, and for patients with a previous Caesarean delivery (CD) (4-6). In Kenya, the guidelines on ECS for PMTCT are less clear, and ECS is offered to antenatal clinic attendees at Pumwani Maternity Hospital in Nairobi. Therefore, guidelines for mode of delivery counselling and provision for ECS for PMTCT are necessary to ensure appropriate use of this intervention at $\mathrm{PMH}$ and throughout Kenya.

Although there is anecdotal evidence from clinical practice that cultural aversion to caesarean delivery in general may exist among some women in sub-Saharan Africa, a CD acceptability study in Nigeria found that $85 \%$ of antenatal women would consent to the procedure if needed (7). Of note, a recent survey of postnatal HIV-positive women in Nairobi found that patient knowledge of ECS for PMTCT was particularly lacking when compared to knowledge of other PMTCT interventions (8). To date there have been no studies characterising HIV -infected women's mode of delivery decisions, and the rate of use of ECS for PMTCT in sub-Saharan Africa remains unknown.

In this study we interviewed HIV -infected women at PMH to determine utilisation of ECS for PMTCT at Pumwani, understand patient knowledge and attitudes regarding the intervention, and characterise mode of delivery decisions among the mothers.

\section{MATERIALS AND METHODS}

Pumwani Maternity Hospital, where this study was conducted, is the largest maternity hospital in East Africa with 40-60 deliveries per day. As a public hospital of the City Council of Nairobi, Kenya, PMH serves a low socio-economic population and is a referral centre for complicated obstetric cases.

The main data collection tool of this study was a structured questionnaire assessing women's sociodemographic characteristics, medical and obstetric history, attitudes and decision making process regarding mode of delivery, and knowledge about ECS for PMTCT. Markers like cell phone ownership and type of cooking fuel were used as proxy indicators for income. At the conclusion of the questionnaire, study participants were given information regarding mode of delivery options for HIV-infected women, including ECS for PMTCT. Benefits as well as risks and discomforts of the procedure, especially in a resource-poor setting like Pumwani, were carefully explained and additional questions regarding ECS acceptability were asked.

All postpartum women at $\mathrm{PMH}$ over the age of 18 years diagnosed with HIV antenatally were invited to participate. Care was taken to ensure patient comfort and privacy during theinterview and sessions were timed postpartum according to mode of delivery. Written informed consent was obtained and confidentiality was maintained by removing identifiable patient information from survey data. Generally, interviews were conducted in Kiswahili. Ethical approval was obtained from Yale University Human Investigations Committee, Kenyatta National Hospital Ethics and Research Committee, and Pumwani Maternity Hospital.

Data were analysed using SPSS-PC version 13.0 (Chicago, IIlinois, USA). The chi-square test was used to compare categorical data and thus determine correlates of mode of delivery decisions and ECS for PMTCT knowledge and attitudes. Adjusted odds ratios were calculated by bivariate logistic regression.

\section{RESULTS}

Interviews were conducted daily over a twelve week period from January to March 2007, and 250 women were enrolled in the study. Three women refused consent and one mother missed invitation to enroll due to record keeping error. All other women diagnosed with HIV antenally who delivered at PMH during this time period participated in the study.

In general, HIV infected mothers delivering at PMH were of low socio-economic status (Table 1). Most werehousewives orjobless (54.4\%) with primary education or less (52.8\%) living in single room homes $(71.2 \%)$, often in one of Nairobi ‘s slums (41.6\%). All women in this study received some form of antenatal care with a median number of four clinic visits (range: $1-17)$, though most women attended small satellite city council clinics around Nairobi $(71.2 \%)$ rather than Pumwani's antenatal clinic. 
Table 1

Study population characteristics

\begin{tabular}{|c|c|}
\hline Characteristic & $\begin{array}{c}\text { No. }(\%) \text { or Median } \\
\text { (Range) }\end{array}$ \\
\hline Median age & $26(18-42)$ \\
\hline \multicolumn{2}{|l|}{ Marital status } \\
\hline Married & $196(78.4)$ \\
\hline Single & $26(10.4)$ \\
\hline Separated/widowed & $28(11.2)$ \\
\hline \multicolumn{2}{|l|}{ Level of education } \\
\hline Primary or less & $132(52.8)$ \\
\hline Secondary & $93(37.2)$ \\
\hline College/university & $25(10.0)$ \\
\hline \multicolumn{2}{|l|}{ Number of rooms in home } \\
\hline 1 & $178(71.2)$ \\
\hline$>1$ & $72(28.8)$ \\
\hline \multicolumn{2}{|l|}{ Cooking fuel } \\
\hline Paraffin/Charcoal/Firewoo & $202(80.8)$ \\
\hline Gas/Electricity & $48(19.2)$ \\
\hline \multicolumn{2}{|l|}{ Type of toilet } \\
\hline Shared & $198(79.2)$ \\
\hline Private & $52(20.8)$ \\
\hline Buys the newspaper & $91(36.4)$ \\
\hline Owns a cell phone & $109(43.6)$ \\
\hline Owns a radio & $204(81.6)$ \\
\hline Owns a television & $146(58.4)$ \\
\hline \multicolumn{2}{|l|}{ Time of HIV diagnosis } \\
\hline During pregnancy & $188(75.2)$ \\
\hline Before pregnancy & $62(24.8)$ \\
\hline \multicolumn{2}{|l|}{ CD4 Count } \\
\hline Median & $340(3-1100)$ \\
\hline Not done & $155(62.0)$ \\
\hline Surgical history & $25(10.0)$ \\
\hline Living children & $179(71.6)$ \\
\hline \multicolumn{2}{|l|}{ Site of antenatal care } \\
\hline \multicolumn{2}{|l|}{ Pumwani } \\
\hline (attended at least once) & $38(15.2)$ \\
\hline City Council Satellite Clin & I78 (71.2) \\
\hline Private Clinic & $34(13.6)$ \\
\hline \multicolumn{2}{|l|}{ ARV regime* } \\
\hline Antenatal AZT only & $6(2.4)$ \\
\hline Intrapartum NVP only & $135(54.0)$ \\
\hline \multicolumn{2}{|l|}{ Antenatal AZT and } \\
\hline Intrapartum NVP & $47(188)$ \\
\hline HAART & $50(200)$ \\
\hline No ARV regimen & $12(48)$ \\
\hline \multicolumn{2}{|l|}{ Outcome } \\
\hline Live birth & $240(91.9)$ \\
\hline Stillbirth/Neonatal death & $21(8.1)$ \\
\hline Average infant weight & 3000 (500-4500) grams \\
\hline
\end{tabular}

The majority of women in the study learned their HIV status during the current pregnancy $(75.2 \%)$, making this a newly-diagnosed population with unique HIV counselling and education needs. Most women did not have an antenatal CD4 count measured (62.0\%) and many received single dose nevirapine (NVP) (54.0\%) as their antiretroviral prophylaxisfor PMTCT. Of women with measured CD4 counts, the median was 340. One visit to Pumwani's Antenatal Clinic was associated with superior HIV care of patients in this study. Women who attended Pumwani were 7.61 (95\% CI: 3.48-16.7, $\mathrm{p}<0.001$ ) more likely to have had CD4 count measured and 2.21 (95\% Cl: $1.10-4.44, \mathrm{p}=0.024)$ more likely to have received the more effective ARV regimens available in Kenya, including zidovudine plus NVP or highly active antiretroviral therapy (HAART). Of note, attendance at PMH Antenatal Clinic was not associated with patient education level or socio-economic markers.

Although $92 \%$ of women in the study had received PMTCT counselling only 36\% (90/250) of the participants had ever heard of ECS for PMTCT. Generally women learned about ECS from doctors or PMTCT counsellors in antenatal clinic $(80.0 \%)$, though $13.3 \%$ of mothers received information from local media, including radio and television. Of the women aware of ECS for PMTCT, most thought it was "very effective" (53.3\%). Few women $(2.4 \%)$ thought incorrectly that elective Caesarean section might actually increase the risk of HIV transmission to the infant.

Knowledge of ECS for PMTCT was correlated with level of patient education on multivariateanalysis. Women with secondary education or higher were 2.69-fold more likely (95\% CI: 1.41-5.14, $\mathrm{p}<0.001$ ) to have heard of the intervention than their less educated counterparts. Diagnosis with HIV before pregnancy was also associated with patient education on ECS for PMTCT (OR=2.31, 95\% CI: 1.17-4.55, p=0.0I6). Finally, women who attended ANC at PMH at least once were 14.28 (95\% CI: 5.44-37.52, $\mathrm{p}<0.001$ ) times more likely to have heard of elective caesarean section than their peers attending other City Council clinics or private clinics.

In an effort to understand women's attitudes towards caesarean delivery in general, all participants were asked open-ended questions about CD regardless of mode of delivery. Table 2 illustrates the mothers' concerns regarding the dangers of Caesarean delivery and data represent the number of women who cited each issue without prompting. Although the most women were worried about increased recovery time and minor complications after CD, about half of the study participants cited death (generally described as a fear of anaesthesiarelated mortality) as a worrisome complication of $\mathrm{CD}$. These data illustrate that most participants were acutely aware of the dangers of Caesarean delivery, even the most serious complications. 
Table 2

Caesarean delivery concerns

\begin{tabular}{lcc}
\hline Patient Concern & No. (\%) \\
\hline Increased recovery time with CD & 128 & 66.3 \\
Increased complications with CD* & 107 & 55.4 \\
Increased risk of death & & \\
with CD/general anaesthesia & & \\
over vaginal delivery & 94 & 48.7 \\
Increased pain with CD & 64 & 33.2 \\
Increased cost with CD & 21 & 10.9 \\
Decreased future fertility with CD & 18 & 9.3 \\
Other & 22 & 8.8 \\
\hline
\end{tabular}

The majority of women in this study planned to deliver vaginally $(72.0 \%)$, though $13.6 \%$ of women planned CD while $14.4 \%$ had no plan regarding mode of delivery (Table 3). Most study participants planned to deliver at PMH (80.4\%) though $18 \%$ of the patients planned to delivery at a secondary health facility such as a City Councilor private clinic. Three women $(1.2 \%)$ planned to deliver at home. Generally women who planned to deliver at clinics were referred for to PMH evaluation of obstetrical complications including poor labor progression or foetal distress.

Women with no delivery plan stated that they had not received counselling regarding mode of delivery or did not think of it prior to the time of delivery. Correlates associated with having a mode of delivery plan on multivariate analysis included being diagnosed with HIV before pregnancy (OR=11.12, 95\% CI: 148-83.90, $\mathrm{p}<0.019)$ and knowledge of ECS for PMTCT $(\mathrm{OR}=3.56$, 95\% Cl: 1.15-11.03, $\mathrm{p}=0.028$ ) (Table 4).

Table 3

Planned and actual mode of delivery

\begin{tabular}{|c|c|c|c|c|c|c|c|}
\hline \multirow{3}{*}{$\begin{array}{l}\text { Planned mode of } \\
\text { delivery }\end{array}$} & \multicolumn{7}{|c|}{ Actual mode of delivery } \\
\hline & \multicolumn{2}{|c|}{ SVD } & \multicolumn{2}{|c|}{ ECS } & Emergent CD & \multicolumn{2}{|c|}{ Total } \\
\hline & No. & $(\%)$ & No. & $(\%)$ & No. $(\%)$ & No. & $(\%)$ \\
\hline SVD $^{*}$ & 145 & 80.6 & 1 & 0.6 & $34 \quad 18.9$ & 180 & 72.0 \\
\hline ECS $^{*}$ & 18 & 52.9 & 7 & 20.6 & 26.5 & 34 & 13.6 \\
\hline No plan & 30 & 83.3 & 2 & 5.6 & $4 \quad 11.1$ & 36 & 14.4 \\
\hline Total & 193 & 77.2 & 10 & 4.0 & $46 \quad 18.4$ & 250 & 100 \\
\hline
\end{tabular}

Table 4

Factors associated with mode of delivery planning

Correlate

Adjusted OR (95\% CI) P-value

Correlates of having a plan for mode of delivery

Diagnosed with HIV before current pregnancy

$11.12(1.48-83.90)$

0.019

Knowledge of ECS for PMTCT

$3.56(1.15-11.03)$

0.028

Correlates of planning ECS for PMTCT

Access to a private toilet

$4.13(1.14-14.94)$

0.031

Surgical history

$36.00(6.32-205.02)$

$<0.001$

Knowledge of ECS for PMTCT

$27.34(5.04-148.20)$

$<0.001$

Attended clinic at Pumwani at least once

$9.12(2.94-28.28)$

$<0.001$

Correlates of delivering by ECS for PMTCT

Planned to deliver by ECS for PMTCT

$19.52(3.69-103.23)$

$<0.001$ 
Nearly all women who planned ECS (33/34) did so for PMTCT purposes although two patients cited placenta previa while two patients cited a history of $\mathrm{CD}$ as secondary indications. One patient planned ECS because she had a history of three previous CD but had not heard of ECS for PMTCT. Of note, eight Caesarean delivery planners $(23.5 \%)$ did not realise that Caesarean section must be performed before labour and rupture of membranes to optimally prevent transmission of the virus to the infant. This illustrates that even among women planning ECS for PMTCT, correct knowledge may be lacking.

On multivariate analysis, planning ECS was not correlated with higher patient education levels, though it was associated with access to a private toilet $(\mathrm{OR}=4.13 ; 95 \% \mathrm{CI}: 1.14-14.94, \mathrm{p}=0.031)$, a marker of higher socio-economicstatus. The strongest independent correlates of ECS planning included having a history of abdominal surgery $(\mathrm{OR}=36.00$, 95\% CI: 6.32-205.00, $\mathrm{p}<0.001)$, attending clinic at PMH (OR=9.12, 95\% CI: 2.94-28.28, $\mathrm{p}<0.001)$, and knowledge of ECS for PMTCT $(\mathrm{OR}=27.34,95 \% \mathrm{Cl}$ 5.04-148.2, $\mathrm{p}<0.001$ ) (Table 4).

The majority of women who planned ECS ended up delivering either vaginally $(52.9 \%)$ or by emergency $\mathrm{CD}(26.5 \%)$ with just $20.6 \%(7 / 34)$ planning and subsequently delivering by ECS (Table 3). Generally this change in delivery plan was caused by labour before scheduled ECS $(55.6 \%, 15 / 27)$ or poor patient education regarding ECS $(29.6 \%, 8 / 27)$. Other reasons for planning and not delivering by ECS included intrauterine foetal death $(1 / 34)$, not being offered ECS upon admission at PMH (1/34), and operating room unavailability $(1 / 34)$. As indicated in Table 4 , the only factor correlated with delivery by elective Caesarean section on multivariate analysis was planning to deliver by ECS (OR $=19.52,95 \%$ CI: 3.69-103.23, $\mathrm{p}<0.001)$. Women who delivered by ECS but planned another mode did so after coming to PMH in false labour, being admitted to the hospital and subsequently counselled on ECS.

All women who delivered by ECS $(10 / 10)$ were very satisfied with their mode of delivery and would strongly recommend this mode of delivery to other HIV -infected women. Spontaneous vaginal delivery (SVD) also proved satisfactory for most study participants with a satisfaction rating of $89.1 \%$ (172/193). Emergent CD proved to be the least satisfying mode of delivery in this study with $40.4 \%$ (19/47) of women feeling unsatisfied with this mode of delivery.

After receiving information on ECS for PMTCT, $48.0 \%(120 / 250)$ of the women in this study would have consented to the procedure if offered to them during this pregnancy or would opt for the procedure in the future. If the cost of ECS for PMTCT was the same as vaginal delivery at PMH, $67.6 \%(169 / 250)$ of the mothers would opt for ECS, indicating that cost represents a significant barrier to choosing this mode of delivery. Correlates of post-counselling acceptability of ECS for PMTCT on multivariate analysis included secondary education $(\mathrm{OR}=2.1$ 5,95\% Cl: 1.16-3.99, $\mathrm{p}<0.001$ ), having living children $(\mathrm{OR}=2.09,95 \%$ CI: 1.12-3.90, $\mathrm{p}=0.023)$ or a surgical history ( $\mathrm{OR}=3.23,95 \% \mathrm{CI}$ : 1.15-9.05, $\mathrm{p}=0.011)$, and attending clinic at $\mathrm{PMH}$ at least once $(\mathrm{OR}=3.06,95 \%$ CI: $1.32-7.13, \mathrm{p}=0.001$ ).

\section{DISCUSSION}

This study represents the first assessment of mode of delivery decisions among HIV-infected women in sub-Saharan Africa. Although there have been numerous studies to date with conflicting results regarding safety of ECS for PMTCT in resource-poor settings, utilisation of this intervention has not been explored or quantified in Africa until now (9-11).

The results of this study indicate that although HIV-infected women of low socio-economic status delivering at a large health facility in Nairobi almost universally receive information on PMTCT, the topic of elective Caesarean section is generally not included in standard counselling. Even women who do receive some education regarding ECS for PMTCT often have inaccurate knowledge of the procedure that may affect delivery of this PMTCT intervention. In addition, women who plan ECS for PMTCT may not ultimately receive this intervention, owing to an incomplete understanding of the intervention as well as lack of uniform procedures for ECS provision at PMH. This gap in patient education and service delivery could be due to unclear policy regarding recommendation of and provision for ECS for PMTCT in Kenya, and possibly other developing countries. We are not advocating for widespread implementation of elective caesarean section in resource-poor settings, but instead aim to highlight the need for clarity on this issue.

As described in the introduction, PMTCT guidelines in Tanzania and Lesotho include recommendation of ECS where $\mathrm{CD}$ may be required for other obstetrical reasons $(5,6)$. Of note, our data illustrate that delivery by elective Caesarean section for PMTCT may be acceptable in this population despite patient awareness of the risks of $\mathrm{CD}$, a finding consistent with past studies of $C D$ acceptability in sub-Saharan Africa (7). A policy of counselling on and utilisation of elective caesarean section targeted specifically at Kenyan women with other indications for CD who find ECS acceptable could promote maximally efficient use of minimal resources. However, patient barriers to utilisation of ECS for PMTCT, including cost, need to be addressed if this intervention is to be used in even limited cases. 
Interestingly, we found that women delivering at PMH do not generally attend ANC at the hospital, but rather receive antenatal care at smaller City Council satellite clinics. In fact attendance of clinic at PMH just one time was associated with superior HIV and antenatal care, suggesting that improvement of PMTCT services at the tertiary care level may not reach most of the patients who require PMTCT services. To ensure optimal counselling and services for HIVinfected mothers in Nairobi, including education on ECS for PMTCT, development and monitoring of PMTCT programmes should begin at the level of the primary care clinic. A policy of referral of HIVinfected pregnant patients (especially ECS candidates) to tertiary hospitals for evaluation is a potential way to improve the care of this high-risk population.

Our study had some limitations. In Nairobi and much of sub-Saharan Africa, many women labour and deliverathomealone or with traditionalbirthattendants. Because we included only women delivering a tertiary care centre, the study findings, including attitudes towards ECS for PMTCT, may not be generalisable to all Kenyan women. Secondly, the number of women planning and actually delivering by ECS for PMTCT was small, limiting the study's power to determine factors associated with planning and delivering by ECS for PMTCT and resulting in the wide confidence intervals (Table 4). Finally, although care was taken to elucidate both risks and benefits of ECS for PMTCT to study participants, itis possiblethat acceptability of ECS for PMTCT amongst antenatal Kenyan women may be different from what we observed in this hypothetical postpartum situation.

This study highlights the disconnect between patient knowledge, attitudes, plans and practices regarding ECS for PMTCT and in turn the lack of uniformity of provision for this PMTCT intervention at a tertiary care centre in Kenya. Further study is needed to elucidate issues of safety, cost, and both patient and institutional barriers to safe and effective implementation of ECS for PMTCT in resourcelimited settings. Clear guidelines on the role of ECS for PMTCT in Kenya and other resource-poor settings are urgently required.

\section{ACKNOWLEDGEMENTS}

To sister P. Omwakwe and the PMTCT counsellors and staff at Pumwani Maternity Hospital. Dr. S. Thung of Yale University, Department of Obstetrics and Gynaecology.
Ethical approval was obtained from Yale University Human Investigations Committee, Kenyatta National Hospital Ethics and Research Committee and Pumwani Maternity Hospital.

This project wassupported by NIHResearch GrantNo. 043 TW000007 funded by the Fogarty International Centre Research Scholars Programme.

\section{REFERENCES}

1. UNAIDS. AIDS epidemic update. Available at www. unaids.org. Retrieved April 16, 2008.

2. Read, J.S. and Newell, M.L. Efficacy and safety of caesarean delivery for prevention of mother to child transmission of HIV - 1. Cochrane Dalahase of Syslemalic Reviews 2005, Issue 4. Art. No: CD005479. DOl: 10/1002/14651858.CD005479.

3. The European Mode of Delivery Collaboration. Elective caesarean section versus vaginal delivery in prevention of vertical transmission: a randomized clinical trial. Lancet. 1999; 353: 1035-1039.

4. WHO, USAID. National Guide to Monitoring and Evaluating Programmes for the Prevention of HIV in Infants and Young Children. 2004.

5. Tanzania Ministry of Health: National Guidelines for Prevention of Mother to Child Transmission of HIV, March 2004.

6. Government of Lesotho: Guidelines to PreventMother to Child Transmission of HIV, June 2004.

7. Awoyinka, B.S., Ayinde, A.O. and Omigbodun, A.O. Acceptability of cesarean delivery to antenatal patients in a tertiary health facility in South-West Nigeria. J. Obstet Gynae. 2006; 26: 208-210.

8. Ong'ech, J.O., Omondi-Ogutu, J., Mchoki, M., et al. Knowledge, attitude, and practice on perinatal HIV transmissionand preventivemeasures among antenatal mothers at Kenyatta National Hospital. J. Obstet. Gyne. Eastern and Cent. Afr. 2006; 19: 1 - 11.

9. Panburana, P., Phaupradit, W., Tantisirin, O, et al. Maternal complications after cesarean section in HIV -infected pregnant women. Aust. New Zealand J. Obst. Gynae. 2003; 43: 160-163.

10. Bjorklund, K., Mutyaba, T., Nabunya, E. and Mirembe, F. Incidence of post cesarean infections in relation to HIV status in a setting with limited resources. Acta. Obstet. Gynecol. Scand. 2005; 84: 967-971.

11. Koigi-Kamau, R., Kabare, L. W. and WanyoikeGichuhi, J. Incidence of wound infection after ceasarean delivery in a district hospital in Central Kenya. East Afr. Med. J. 2005; 82: 357-361. 